\title{
La integración educativa y social del alumnado con discapacidad en el EEES: Universidad de Bolonia
}

\section{The educational and social integration of students with disabilities in the EEES: University of Bologna}

\author{
Antonio LUQUE DE LA ROSA y Rafaela GUTIÉRREZ CÁCERES \\ Universidad de Almería
}

Recibido: Febrero 2013

Aceptado: Abril 2013

\section{Resumen}

En la actualidad, la universidad europea se encuentra ante el reto de promover la efectiva atención al estudiante con discapacidad en el seno de sus instituciones, para lo cual el conjunto de ideas y actitudes de los miembros de la comunidad universitaria es determinante. En esta línea, y continuando investigaciones precedentes en otros países europeos, surge la iniciativa de realizar el presente estudio en un contexto tradicionalmente integrador como es el italiano, analizando las concepciones que el alumnado tiene acerca de la integración y atención a sus compañeros con discapacidad.

Los sujetos investigados fueron 272 alumnos procedentes de la facultad de Ciencias de la Educación de la universidad de Bolonia (Italia). Los resultados se han plasmado a nivel global y en función de unas categorías de análisis que resaltan los principales indicadores de la atención a la discapacidad en la universidad. Al mismo tiempo se ha realizado un análisis comparativo entre subgrupos en función de diversas variables y a partir del conjunto de observaciones planteadas por los encuestados se ha realizado una interpretación cualitativa con el fin de evaluar la influencia del contexto universitario italiano en la percepción de los estudiantes sobre la integración del alumnado con discapacidad.

Tras los análisis realizados, podemos concluir que, en términos generales, los estudiantes participantes de la universidad de Bolonia poseen unas ideas y actitudes favorables hacia la integración educativa y social de los estudiantes con discapacidad. Si bien, a pesar de que los planteamientos institucionales avanzan teóricamente a buen ritmo, la integración del colectivo de estudiantes con discapacidad en la educación superior es una tarea que se muestra aún inconclusa en la Universidad de Bolonia.

Palabras clave: integración educativa, inclusión social, discapacidad, educación superior, actitudes. 


\begin{abstract}
Today, the European university is faced with the challenge of promoting effective care to students with disabilities within their institutions, for which the set of ideas and attitudes of members of the university community is crucial. In this line, and continuing previous research in other European countries, there is the initiative to conduct this study in a context traditionally inclusive as it is the Italian one, analyzing the notions that students have about the integration and attention to their peers with disabilities.

The subjects of the study were 272 students from the Faculty of Education at the University of Bologna (Italy). The results are reflected globally and in terms of categories of analysis that highlight the main indicators of attention to disability in college. At the same time a comparative analysis of subgroups has been made according to different variables and from the issues raised by the respondents a qualitative interpretation was carried out in order to evaluate the influence of Italian university context in the perception of students about integration of students with disabilities.

After the analyses, we conclude that, overall, participating students from the University of Bologna have some favoral ideas and attitudes towards education and social integration of students with disabilities. Nevertheless, even though institutional approaches are advancing at a good pace in theory, collective integration of disabled students in higher education is a task that is still to be concluded at the University of Bologna.
\end{abstract}

Keywords: integration, social inclusion, disability, higher education, attitudes.

En el entorno europeo, como en el resto de los sistemas educativos, la universidad ha sido tradicionalmente el nivel educativo más segregador y excluyente con respecto a la educación del alumnado con discapacidad (Díaz, 2000), hecho que contrasta con la corriente integradora que se ha venido desarrollando a partir de los años ochenta en los niveles no universitarios.

Sin embargo, con el paso del tiempo, se han ido instaurando en las universidades europeas mecanismos que facilitan el acceso de este tipo de alumnado a dichas instituciones, viéndose incrementado considerablemente el número de alumnos con discapacidad que ingresan en la educación superior (Eches y Ochoa, 2005; Granados, 2000; Konur, 2006).

De esta manera, actualmente, en las instituciones de educación superior se desarrollan una serie de acciones de asesoramiento y apoyo a la comunidad universitaria que contribuyen a suprimir barreras para el acceso al currículo y facilitan el fomento de la participación e inclusión social de los universitarios con discapacidad (Arana, Garcia y Rodriguez, 2008; Bausela, 2004; Center y Ward, 1987; Clough y Lindsay, 1991; Rubiralta, 2012).

Este hecho se ha reflejado en la organización y el funcionamiento de las universidades, promoviendo que en todos los países europeos se estén adaptando dichos centros en su estructura y actuación a los principios de normalización, integración e inclusión y concibiendo la educación como un servicio que debe ser prestado al conjunto de la ciudadanía (Mangas, 2005). 
Sin embargo, en esta implantación de la atención a la diversidad a nivel universitario, se están produciendo diferencias de matiz entre los diversos países europeos, similares a las que se produjeron con anterioridad a la hora de promover la integración en el resto de los niveles educativos. Es por eso que en esta investigación hemos decidido profundizar en uno de los contextos europeos que destacaron por su carácter integrador en la escuela ordinaria en los años ochenta (Italia), al igual que podemos citar otros como España, Reino Unido, Suecia y Dinamarca.

Como hemos comentado, desde hace mucho tiempo Italia se ha reconocido como líder en la promoción de un sistema educativo nacional con carácter inclusivo. Así, hacia mediados de los años setenta, surgió con fuerza en este país el desarrollo de políticas sociales integradoras repercutiendo de forma progresiva y generalizada en todo el sistema educativo, en concreto a partir de la promulgación de la Ley $n^{\circ} 517$ del 4 de agosto de 1977.

Posteriormente, con la aprobación de la Ley $\mathrm{n}^{0} 104$ del 5 de febrero de 1992 modificada por la Ley 17/1999-, se establece el derecho a la educación de todas las personas con discapacidad no sólo en el ámbito escolar sino también en el contexto universitario (art. 12). Con dicha norma se intenta garantizar a los estudiantes universitarios con discapacidad la provisión de materiales técnicos y didácticos, servicios de tutoría especializada (tutoratto specializzato), recursos adaptados en los exámenes y la presencia de un docente con funciones de coordinación, seguimiento y apoyo en todas las iniciativas y propuestas para la integración en el ámbito universitario.

Asimismo, en consonancia con los postulados teóricos que se iban generando desde los noventa, el establecimiento de esta ley refuerza la atención a la discapacidad en todo el sistema educativo italiano, incidiendo incluso en la misma definición de "discapacidad" al otorgarle ahora un carácter más inclusivo que en los años setenta, lo cual refleja la madurez de una nueva conciencia educativa que en España se vio ralentizada a nivel normativo (Sciacca, Sciuto, Signorino, Venuto y Nicotra, 2008). Así, "una persona discapacitada es la que tiene problemas físicos, psíquicos o sensoriales, de forma estable o progresiva; esto le provoca dificultades en el aprendizaje, en las relaciones o integración laboral hasta el punto de determinar desventajas sociales o de marginación" (Ley n. 104 del 5 de febrero del 1992, art.3).

Si analizamos el contenido de dicha norma, se evidencia la concepción de la discapacidad como el hecho de encontrarse ante una situación de desventaja que demanda las consecuentes ayudas (Trainito, 2003). Por otra parte, se omite la expresión de portador de hándicap, términos utilizados en anteriores normativas, desmontando su carácter obstaculizador e inabarcable y valorando más a la persona en su unidad, unicidad e integridad (De Mauro, 2004).

Así, esta ley marca un avance normativo muy importante en la temática que nos ocupa, al declarar que el derecho a la educación no puede ser obstaculizado por dificultades en el aprendizaje ni por otras situaciones derivadas de la discapacidad (Pavone, 2007), promulgando que la integración escolar tiene como objetivo el 
desarrollo de las capacidades de la persona discapacitada en el aprendizaje, en la comunicación y en las relaciones sociales (Ley n. 104 del 5 de febrero del 1992).

Desde entonces se vienen realizando diversas experiencias impulsoras de la innovación en el sistema educativo italiano, claves para el cambio a nivel social, existiendo una tendencia creciente en cuanto a la matriculación de estudiantes con discapacidad en las instituciones italianas de educación superior.

Sin embargo, a pesar de su vertiente claramente integradora, la mayoría de las personas con discapacidad en dicho país consiguen niveles educativos inferiores respecto al resto de la población. Así, son escasos los alumnos que acceden a la universidad para cursar los estudios correspondientes. Respecto al grupo de personas con discapacidad, sólo el 2,55\% tienen alguna titulación universitaria de grado o posgrado; debiendo señalar que el $49,88 \%$ de las personas con discapacidad alcanzan sólo el nivel de educación primaria, no consiguiendo superar ningún nivel instructivo el 22,83\% del colectivo (De Anna, 2010).

En este sentido, el proceso de integración en Italia no está igualmente exento de críticas, reflejo de factores como: una escasa financiación y colaboración entre los organismos públicos y entre éstos y la sociedad, una insuficiente información y sensibilización por parte de los miembros universitarios acerca de la discapacidad y sus implicaciones en el aprendizaje y desarrollo de la persona, una inadecuada formación por parte de los maestros especialistas de apoyo didáctico, la insuficiente capacitación dirigida al profesorado no especialista... (De Anna, 2010).

Una de las principales barreras para la inclusión del alumnado en el aula y para la atención a la diversidad del alumnado en el proceso de enseñanza-aprendizaje se identifica en la actitud y pensamiento del propio profesorado y resto de la comunidad educativa.

Son muchos los autores que conciben las ideas y actitudes hacia las personas con discapacidad como un aspecto fundamental en su éxito en los procesos de integración y en su proceso de enseñanza-aprendizaje (Bueno, 2010; Castejón, 2004; Jarvis y French, 1990; Verdugo y otros, 1994).

Si nos referimos al agente que interactúa con las personas con discapacidad y sus concepciones y actitudes hacia dicho colectivo, existen varias investigaciones que destacan como uno de los elementos claves para poder llevar a término la inclusión en el aula, la adquisición del compromiso de los profesores (Ainscow, 2001; Stainback y Stainback, 1999). Los docentes han de tener la oportunidad de formarse y sensibilizarse para comprender las ventajas del cambio hacia la cultura inclusiva, siendo necesaria dicha actitud de aceptación y disposición en cualquier proceso de reforma educativa y social.

Con relación al hecho de las ideas y actitudes en el colectivo de alumnado sin discapacidad hacia estudiantes con discapacidad encontramos similares aportaciones en los estudios realizados por Dendra, Durán y Verdugo (1991) referidas a la integración de escolares con necesidades educativas especiales o en Arias (1994), Sáenz (1990) y Verdugo, Arias y Jenaro (1994) referidas a la integración de discapacitados en general. 
Si hacemos referencia a la etapa educativa que cursan, son muchos los estudios que se han realizado sobre las aulas inclusivas a nivel de educación primaria y secundaria (Hegarty, 1988; Stainback y Stainback, 1999; Rosenvinge, 1991; Vega, 2000), pero no ocurre lo mismo a nivel universitario, -véase, por ejemplo, la revisión realizada por Avramidis, Bayliss y Burden (2000)-.

Así, podemos reseñar, entre otras, las realizadas por Gómez e Infante (2004) que estudian concepciones y actitudes de estudiantes universitarios hacia personas con discapacidad y minorías étnicas; Suriá (2011) que realiza un análisis comparativo sobre las actitudes de los estudiantes hacia sus compañeros con discapacidad; o estudios como los llevados a cabo por Sánchez (2009) en Almería (España) o Guzmán y Sánchez (2011) en Tlaxcala (México), analizando las ideas y actitudes de los diversos sectores de la comunidad educativa (incluidos los estudiantes sin discapacidad) hacia el colectivo del alumnado con necesidades educativas especiales en países diversos en cuanto a desarrollo económico y cultura inclusiva.

En este sentido, con el presente estudio pretendemos complementar sendas investigaciones previas en las que participamos como parte del equipo investigador en el ámbito de la universidad de Almería y Tlaxcala, contrastando los resultados allí alcanzados con los obtenidos en Bolonia (Italia). Un país europeo que, al igual que España, posee una larga trayectoria en la inclusión educativa, en el cual, tras una primera fase "ideológica" de la inclusión educativa, se ha adoptado la decisión de implementar dicha filosofía en la estructura y funcionamiento de las instituciones universitarias, lo cual requiere del apoyo de una investigación rigurosa y crítica contrastando con otras experiencias con el fin de reflexionar, profundizar y mejorar la calidad de dichas prácticas educativas.

Por todo lo expuesto y ante la ausencia de estudios realizados en el contexto italiano en relación con la educación superior, surge la necesidad de realizar la presente investigación cuya finalidad se centra en el análisis de la situación actual de la integración educativa y social de los estudiantes con discapacidad, desde la perspectiva de los estudiantes de la universidad de Bolonia avanzando así en el conocimiento de las características de dicha realidad en el Espacio Europeo de Educación Superior.

\section{Material y métodos}

Atendiendo a los supuestos teóricos previos, y siguiendo la línea de investigación descrita en el apartado anterior, los objetivos específicos que planteamos son los siguientes:

- Conocer las ideas que manifiestan los estudiantes sobre los aspectos favorecedores de la integración educativa y social del alumnado con discapacidad en la Universidad de Bolonia.

- Estudiar las opiniones que declaran dichos estudiantes sobre los obstáculos que dificultan la integración universitaria del alumnado con discapacidad en la actualidad. 
- Indagar las actitudes que poseen estos estudiantes hacia el alumnado con discapacidad y su integración en la Universidad de Bolonia.

- Examinar las opiniones y actitudes de los citados estudiantes según las variables: agrupación de edad, sexo y experiencia sobre integración del alumnado con discapacidad en la universidad.

Teniendo en cuenta los objetivos específicos planteados, hemos desarrollado una investigación enmarcada dentro de una metodología combinada dada la complejidad característica de la realidad educativa que indagamos, de manera que hemos utilizado, por un lado, un enfoque caracterizado por la recogida y el análisis cuantitativo de datos relativos al objeto de estudio; mientras que por otro lado, hemos adoptado un diseño de tipo descriptivo desde el paradigma naturalista, teniendo en cuenta la necesidad de realizar un análisis en y desde la propia práctica en la que se desarrolla la atención educativa a estudiantes en la Universidad de Bolonia.

En coherencia con el diseño metodológico adoptado y en función de los objetivos específicos planteados en esta investigación, la muestra se ha obtenido de forma incidental u ocasional aprovechando la colaboración investigadora de uno de los autores en la estancia desarrollada en el presente curso en Bolonia, siendo esta muestra un agrupamiento natural integrado por los estudiantes que voluntariamente colaboraron en la cumplimentación de los cuestionarios.

Así, en este estudio participaron 272 alumnos procedentes de la facultad de Ciencias de la Educación de la universidad de Bolonia (Italia), que se encuentran matriculados en la titulación universitaria correspondiente al grado de Ciencias de la Educación Primaria, siendo el $82 \%$ de los encuestados, alumnos que se encuentran en el $2^{\circ}$ curso. La media general en cuanto a edad es 22,46 años (SD: 4,59; Rango: 19-41 años), siendo el $88,9 \%$ alumnos que poseen una edad comprendida entre los 19 y los 26 años. En relación con el género, predominan las chicas con un $94,4 \%$. De entre ellos, un total de 41 estudiantes afirman que tienen o han tenido en sus clases a compañeros que presentan una discapacidad mayoritariamente de tipo físico y psíquico, siendo sólo 19 de ellos los que manifiestan que han recibido en la universidad algún tipo de información en relación con la atención a alumnos con discapacidad.

Para la obtención de datos hemos utilizado como técnica de investigación la encuesta, habiéndose aplicado un cuestionario a la muestra señalada de estudiantes de la universidad de Bolonia, con el fin de conocer la percepción que éstos poseen sobre la integración del alumnado con discapacidad (véase Anexo 1) ${ }^{1}$.

Dicho instrumento nos ha permitido recoger abundante y suficiente información de manera rápida y con costos materiales y humanos bajos. La investigación realizada a través de una encuesta implica la obtención de información directamente de un grupo

\footnotetext{
${ }^{1}$ Dicho instrumento de recogida de datos ha sido elaborado atendiendo a las necesidades específicas del contexto italiano, realizando para ello una adaptación pertinente a partir del cuestionario sobre ideas y actitudes ante la integración educativa y social de los estudiantes con discapacidad, que ha sido extraído de Sánchez Palomino (2009).
} 
de individuos, cuya finalidad es descriptiva, además de permitir obtener estimaciones generales de los aspectos objeto de estudio.

Se trata de una técnica muy extendida en la investigación social, mediante la que se obtienen datos a través de cuestionarios de opinión aplicados a un grupo de personas (Rodríguez, Gil y García, 1996; Goetz y LeCompte, 1998), permitiendo su transcripción a valores numéricos $\mathrm{y}$, así, operar estadísticamente con ellos para describir los resultados.

La fiabilidad de dicho cuestionario se ha calculado a partir de los resultados obtenidos en este estudio utilizando el índice de consistencia interna. Ha resultado ser alta, ya que el coeficiente alfa de Cronbach, en todas las dimensiones, fue superior a 0,65 , siendo igual a 0,80 (valor alfa $=0,805$ ). El análisis de los enunciados ha mostrado individualmente que son ítems capaces de discriminar entre las distintas opiniones de los encuestados (desviaciones típicas altas) y medias relativamente centradas en el cuestionario (de 1 a 4 valores).

Con el fin de facilitar el análisis e interpretación de los resultados obtenidos, atendiendo a los supuestos teóricos previamente expuestos en el apartado anterior y siguiendo el procedimiento de validación mediante el sistema de "juicio de expertos" (Fox, 1981; Hodder, 2000), con un acuerdo inter-jueces del 97\%, hemos valorado y clasificado los ítems que integran el cuestionario, asignándolos a las correspondientes categorías de análisis o dimensiones, como se muestran en la tabla 1.

\begin{tabular}{|c|c|c|}
\hline CATEGORÍAS & DEFINICIÓN CATEGORÍAS & ITEMS \\
\hline $\begin{array}{l}\text { Adaptaciones } \\
\text { curriculares }\end{array}$ & $\begin{array}{l}\text { Ideas y actitudes que tienen los estudiantes sobre } \\
\text { las adaptaciones curriculares para el alumnado con } \\
\text { discapacidad de la Universidad de Bolonia. }\end{array}$ & $\begin{array}{l}1,3,7,9,15 \\
20,24,25,26 \\
34\end{array}$ \\
\hline Aceptación & $\begin{array}{l}\text { Ideas y actitudes que poseen los estudiantes hacia } \\
\text { el alumnado con discapacidad y su integración en la } \\
\text { Universidad de Bolonia. }\end{array}$ & $\begin{array}{c}2,4,5,6,17 \\
21,23,29\end{array}$ \\
\hline $\begin{array}{l}\text { Formación de los } \\
\text { miembros de la } \\
\text { comunidad } \\
\text { universitaria }\end{array}$ & $\begin{array}{l}\text { Ideas y actitudes que tienen los estudiantes sobre } \\
\text { los aspectos relativos a la formación que reciben los } \\
\text { miembros de la comunidad universitaria para la } \\
\text { integración del alumnado con discapacidad. }\end{array}$ & $\begin{array}{c}8,10,12,32 \\
35,36,37,39\end{array}$ \\
\hline $\begin{array}{r}\text { Servicios } \\
\text { universitarios }\end{array}$ & $\begin{array}{l}\text { Ideas y actitudes que tienen los estudiantes sobre } \\
\text { los servicios universitarios para favorecer la } \\
\text { integración del alumnado con discapacidad. }\end{array}$ & $\begin{array}{l}11,13,14,16 \\
18,19,22,27 \\
28,30,31,33 \\
38,40\end{array}$ \\
\hline
\end{tabular}

Tabla 1. Categorías de análisis y asignación de ítems

Para proceder al análisis de los datos con carácter meramente descriptivo, se han transformado los cuatro niveles de respuesta a los ítems del cuestionario en puntuaciones numéricas, así "totalmente en desacuerdo" se corresponde con una puntuación de 0, "en desacuerdo" con 1, "de acuerdo" con 3 y "totalmente de acuerdo" con 4. Asimismo hemos establecido dos valores con el fin de dicotomizar las cuatro opciones de respuesta: a) rechazo: que engloba las respuestas 1 (totalmente en 
desacuerdo) y 2 (en desacuerdo); b) aceptación: que se refiere a las respuestas 3 (de acuerdo) y 4 (totalmente de acuerdo).

Así, en el análisis de los datos obtenidos hemos utilizado estadísticos descriptivos tales como la distribución de frecuencias, el porcentaje, la media y la desviación típica. De igual manera, considerando los ítems en su sentido propiamente ordinal, hemos aplicado la prueba U de Mann-Whitney, estadístico no paramétrico que compara los grupos independientes que se van estableciendo según la variable nominal considerada (agrupación de edad; sexo; si han tenido o tienen compañeros con discapacidad; si han recibido o no información sobre la presencia de compañeros con discapacidad en el aula).

Finalmente, tras el análisis de las observaciones aportadas por el alumnado, hemos realizado una interpretación cualitativa con el fin de evaluar la influencia del contexto universitario italiano en la percepción de los estudiantes sobre la integración del alumnado con discapacidad en el Espacio Europeo de Educación Superior en Italia.

\section{Resultados: análisis e interpretación}

\section{Análisis descriptivo general}

Si procedemos a analizar globalmente los resultados obtenidos, podemos apreciar que la puntuación media general es de 2,72, lo cual indica una aceptación considerable del hecho integrador en la comunidad de estudiantes universitarios (aproximándose a la respuesta "de acuerdo"), con un margen moderado de variación en las respuestas ( $S D$ : $0,77)$.

Como ítems destacados, señalamos el 23 , en el cual un $94,4 \%$ de los encuestados manifiesta estar de acuerdo o muy de acuerdo con el hecho de que la presencia en las aulas de estudiantes con discapacidad no produce un descenso del nivel académico generalizado $(M: 3,42 ; S D: 0,59)$. En la misma línea encontramos que, según los resultados del ítem 6 , el $90,2 \%$ de los sujetos considera estar de acuerdo o muy de acuerdo con que la existencia en las aulas de estudiantes con discapacidad no dificulta su propio aprendizaje $(M: 3,33 ; S D: 0,69)$.

Similar tendencia hemos observado con relación al ítem 17 (la presencia en las aulas de estudiantes con discapacidad no supone problemas y dificultades), al detectar que el $84,7 \%$ de los encuestados aceptan la idea que en éste se propone $(M: 3,22 ; S D$ : 0,73). Es también destacable cómo el 88,9\% (ítem 5) del alumnado expresa que le gusta relacionarse con compañeros discapacitados a los que pueda serles útil y ayudarles $(M: 3,27 ; S D: 0,69)$. 


\begin{tabular}{|c|c|c|c|c|c|c|c|c|c|c|c|c|c|}
\hline Ítems & 1 & 2 & 3 & 4 & $\mathbf{M}$ & SD & Ítems & 1 & 2 & 3 & 4 & $\mathbf{M}$ & SD \\
\hline 1 & 0.0 & 15,3 & 68,1 & 16,7 & 3,01 & $\mathbf{0 , 5 6}$ & 21 & 0,0 & 13,9 & 48,6 & 37,5 & 3,23 & 0,68 \\
\hline 2 & 6,9 & 9,7 & 41,7 & 41,7 & 3,18 & $\mathbf{0 , 8 7}$ & 22 & 11,1 & 27,8 & 45,8 & 15,3 & 2,65 & 0,68 \\
\hline $\begin{array}{c}3 \\
\text { rec }^{2} \\
\end{array}$ & 38,9 & 44,4 & 13,9 & 2,8 & 1,80 & $\mathbf{0 , 7 8}$ & $\begin{array}{c}23 \\
\text { rec }\end{array}$ & 0,0 & 5,6 & 47,2 & 47,2 & 3,41 & $\mathbf{0 , 5 9}$ \\
\hline $\begin{array}{c}4 \\
\text { rec } \\
\end{array}$ & 2,8 & 34,7 & 41,7 & 20,8 & 2,80 & $\mathbf{0 , 7 9}$ & 24 & 6,9 & 16,7 & 55,6 & 20,8 & 2,90 & $\mathbf{0 , 8 0}$ \\
\hline 5 & 1,4 & 9,7 & 48,6 & 40,3 & 3,27 & 0,69 & 25 & 5,6 & 30,6 & 51,4 & 12,5 & 2,70 & 0,75 \\
\hline $\begin{array}{c}6 \\
\text { rec }\end{array}$ & 1,4 & 8,3 & 45,8 & 44,4 & $\mathbf{3 , 3 3}$ & 0,69 & 26 & 1,4 & 11,1 & 63,9 & 23,6 & 2,70 & $\mathbf{0 , 6 3}$ \\
\hline 7 & 9,7 & 29,2 & 50,0 & 11,1 & 2,62 & $\mathbf{0 , 8 1}$ & 27 & 1,4 & 29,2 & 62,5 & 6,9 & 2,75 & $\mathbf{0 , 5 9}$ \\
\hline 8 & 5,6 & 16,7 & 51,4 & 26,4 & 2,98 & $\mathbf{0 , 8 1}$ & 28 & 2,8 & 30,6 & 56,9 & 9,7 & 2,73 & 0,67 \\
\hline 9 & 8,3 & 36,1 & 48,6 & 6,9 & 2,54 & $\mathbf{0 , 7 4}$ & 29 & 4,2 & 18,1 & 62,5 & 15,3 & 2,73 & $\mathbf{0 , 7 0}$ \\
\hline 10 & 34,7 & 41,7 & 19,4 & 4,2 & 1,93 & $\mathbf{0 , 8 4}$ & 30 & 12,5 & 29,2 & 44,4 & 13,9 & 2,59 & $\mathbf{0 , 8 8}$ \\
\hline 11 & 9,7 & 23,6 & 58,3 & 8,3 & 2,65 & $\mathbf{0 , 7 7}$ & 31 & 1,4 & 22,2 & 59,7 & 16,7 & 2,91 & 0,66 \\
\hline 12 & 23,6 & 34,7 & 36,1 & 5,6 & 2,23 & $\mathbf{0 , 8 8}$ & 32 & 6,9 & 19,4 & 59,7 & 13,9 & 2,91 & 0,76 \\
\hline 13 & 9,7 & 23,6 & 58,3 & 8,3 & 2,65 & $\mathbf{0 , 7 7}$ & 33 & 11,1 & 19,4 & 50,0 & 19,4 & 2,77 & $\mathbf{0 , 8 9}$ \\
\hline 14 & 8,3 & 18,1 & 56,9 & 16,7 & 2,81 & $\mathbf{0 , 8 1}$ & 34 & 25,0 & 27,8 & 22,2 & 25,0 & 2,47 & 1,12 \\
\hline $\begin{array}{c}15 \\
\text { rec }\end{array}$ & 5,6 & 33,3 & 44,4 & 16,7 & 2,72 & $\mathbf{0 , 8 0}$ & 35 & 18,1 & 45,8 & 33,3 & 2,8 & 2,20 & 0,76 \\
\hline 16 & 8,3 & 26,4 & 55,6 & 9,7 & 2,66 & 0,76 & 36 & 8,3 & 19,4 & 51,4 & 20,8 & 2,84 & $\mathbf{0 , 8 5}$ \\
\hline $\begin{array}{c}17 \\
\text { rec }\end{array}$ & 1,4 & 13,9 & 45,8 & 38,9 & 3,22 & $\mathbf{0 , 7 3}$ & 37 & 6,9 & 13,9 & 58,3 & 20,8 & 2,93 & 0,79 \\
\hline 18 & 13,9 & 29,2 & 48,6 & 8,3 & 2,51 & $\mathbf{0 , 8 3}$ & 38 & 20,8 & 13,9 & 47,2 & 18,1 & 2,62 & 1,01 \\
\hline 19 & 5,6 & 40,3 & 48,6 & 5,6 & 2,54 & 0,69 & 39 & 22,2 & 18,1 & 52,8 & 6,9 & 2,44 & 0,91 \\
\hline $\begin{array}{c}20 \\
\text { rec }\end{array}$ & 1,4 & 58,3 & 29,2 & 11,1 & 2,50 & $\mathbf{0 , 7 1}$ & 40 & 5,6 & 31,9 & 47,2 & 15,3 & 2,72 & 0,79 \\
\hline
\end{tabular}

Tabla 2. Resultados descriptivos generales

\footnotetext{
${ }^{2}$ Algunos ítems formulados en sentido negativo se han recodificado al objeto de dar un sentido positivo a todos y cada uno de ellos, de manera que se facilite los análisis posteriores.
} 
En contraste con estas manifestaciones favorables hacia la integración de estudiantes con discapacidad en el ámbito de la educación superior, observamos que el $83,3 \%$ del alumnado encuestado considera que a sus compañeros con discapacidad de la universidad de Bolonia les cuesta más terminar una carrera al no disponer de medios adaptados (ítem $3 ; M: 1,80 ; S D: 0,78$ ).

De la misma manera, según el ítem 35, solamente el 36,1\% expresa estar de acuerdo o muy de acuerdo con que los planes de estudio posean un considerable número de créditos para formar a los maestros en la atención a las discapacidades en el ámbito escolar (M: 2,20; $S D$ : 0,76). Resulta igualmente llamativo que el $58,2 \%$ opine que no se le informa acerca de la presencia en sus aulas de compañeros con discapacidad (ítem 12; $M: 2,23 ; S D: 0,88)$.

Por último, podemos señalar que atendiendo a los valores de la desviación típica, los ítem 1 (se facilita el acceso de los estudiantes con discapacidad a la Universidad) y 21 (la presencia en las aulas de estudiantes con discapacidad beneficia a todos y a todas) destacan en cuanto a la escasa dispersión de resultados con relación a la media obtenida $(S D: 0,56$ y 0,68 , respectivamente), coincidiendo la mayoría de los encuestados en la aceptación de dichos enunciados.

\section{Análisis según categorías de análisis}

A continuación se muestra el análisis en función de la agrupación categorial de los ítems según la asignación anteriormente expuesta en relación con las dimensiones relativas a la integración de los estudiantes con discapacidad en la universidad:

\section{A) Adaptaciones}

En relación con esta categoría se ha encontrado que la puntuación media general es de 2,59 (SD: 0,77), siendo éste un valor numérico que se aproxima a la respuesta "de acuerdo". En el conjunto de los ítems referidos a la dimensión "adaptaciones" se aprecia una valoración media más elevada en el ítem 1, siendo el 84,8\% del alumnado el que opina que se facilita el acceso de los estudiantes con discapacidad a la Universidad. En coherencia con esta afirmación, se ha observado también que el 76,4\% considera que estos estudiantes tienen las mismas oportunidades y posibilidades de promoción que los demás universitarios (ítem 24) y, además, el $87,5 \%$ cree que la titulación alcanzada por parte de éstos supone el garante del conjunto de competencias para su desarrollo profesional (ítem 26). No obstante, cuando se les preguntó si a los estudiantes con discapacidad les cuesta más terminar una carrera por no disponer en la Universidad de los medios adecuados a sus necesidades, curiosamente el 83,3\% de los encuestados contestan afirmativamente estar conforme (ítem 3).

B) Aceptación

En cuanto a la categoría "aceptación" entendida ésta como uno de los requisitos básicos para la inclusión universitaria de los estudiantes con discapacidad, la media general es de 3,14 (SD: 0,71) siendo esta puntuación coincidente con la respuesta "de acuerdo". Se puede destacar cómo en el conjunto de los ítems correspondientes a esta 
categoría, son el 23 y el 6 los que especialmente destacan superando el valor promedio ( $M: 3,41$ y 3,33; $S D: 0,59$ y 0,69, respectivamente). Así, el 94,4\% del alumnado afirma que la presencia de los estudiantes con discapacidad en el aula universitaria no afecta negativamente al nivel académico general (ítem 23). De igual modo, el 90,2\% opina que esta situación no supone ningún obstáculo para el aprendizaje del alumnado (ítem $6)$.

Es preciso señalar que es el ítem 29 el menos valorado positivamente, siendo sólo el $77,8 \%$ quien considera la existencia de una adecuada comunicación entre los estudiantes con discapacidad y sus compañeros, entendida ésta como uno de los apoyos esenciales para su integración en la universidad. En coherencia con esta afirmación se ha encontrado que el $62,5 \%$ del alumnado cree que no tiene dificultad para relacionarse y comunicarse con los estudiantes con discapacidad (ítem 4).

\section{C) Formación}

En referencia a esta categoría se ha obtenido un valor promedio de 2,55 (SD: 0,82), siendo el ítem 8 el más destacado en su puntuación media de modo que el $77,8 \%$ del alumnado considera que se promueve el desarrollo de actitudes positivas hacia los estudiantes con discapacidad y su integración en la universidad. En el mismo sentido, se ha encontrado que el 79,1\% opina que la formación inicial del docente contempla la atención educativa a alumnos con discapacidad (ítem 37) y, además, el 73,6\% cree que se impulsa una concienciación en el ámbito educativo para facilitar servicios adaptados (ítem 32). A pesar de ello, es necesario resaltar que sólo el 23,6\% del alumnado afirma que ha recibido algún tipo de formación necesaria para favorecer la integración de los estudiantes con discapacidad en la universidad (ítem 10).

\section{D) Servicios}

Por último, con respecto a la categoría "servicios" la puntuación media general es de 2,68 (SD: 0,77), un valor muy próximo a la respuesta "de acuerdo". Especialmente relevante es el ítem 31 en la medida en que el $76,4 \%$ de los encuestados piensa que en la Universidad se cumplen las normativas que contemplan los derechos y deberes de los estudiantes con discapacidad. Asimismo, el $73,6 \%$ cree que existen medios disponibles para que estos estudiantes se integren activamente en el ámbito universitario (ítem 14). Pero según el 43,1\% del alumnado, no existe ninguna normativa específica sobre la atención de los estudiantes con discapacidad en la Universidad de Bolonia (ítem 18). Además, no se disponen de los medios suficientes para el desarrollo de adaptaciones en el acceso al currículum (ítem 19).

\section{Análisis comparativos entre subgrupos}

En este subapartado se expone un análisis comparativo de los resultados obtenidos en función de determinados subgrupos de sujetos según las siguientes variables: grupos de edad, sexo y experiencia sobre integración del alumnado con discapacidad en la universidad (información sobre discapacidad y haber tenido compañeros/as con discapacidad). 


\begin{tabular}{|c|c|c|c|c|c|c|c|c|c|}
\hline \multirow[t]{2}{*}{ Ítem } & \multicolumn{2}{|c|}{ Sexo } & \multicolumn{2}{|c|}{$\begin{array}{l}\text { Información } \\
\text { discapacidad }\end{array}$} & \multicolumn{3}{|c|}{ Edad (agrupado) } & \multicolumn{2}{|c|}{$\begin{array}{l}\text { Compañeros } \\
\text { discapacidad }\end{array}$} \\
\hline & Hombre & Mujer & No & Sí & $<=21$ & $22-26$ & $27-41$ & No & Sí \\
\hline 1 & 2,75 & 3,03 & 3,00 & 3,20 & 3,04 & 2,93 & 3,00 & 2,98 & 3,18 \\
\hline 2 & 3,00 & 3,19 & 3,19 & 3,00 & 3,18 & 3,00 & 3,50 & 3,16 & 3,27 \\
\hline 3 rec & 1,75 & 1,81 & 1,84 & 1,40 & 1,76 & 2,13 & 1,50 & 1,77 & 2,00 \\
\hline 4 rec & 2,25 & 2,84 & 2,85 & 2,20 & 2,76 & 2,87 & 3,00 & 2,82 & 2,73 \\
\hline 5 & 3,50 & 3,26 & 3,27 & 3,40 & 3,22 & 3,27 & 3,63 & 3,34 & 2,91 \\
\hline $6 \mathrm{rec}$ & 3,25 & 3,34 & 3,31 & 3,60 & 3,31 & 3,53 & 3,13 & 3,36 & 3,18 \\
\hline 7 & 3,00 & 2,60 & 2,66 & 2,20 & 2,49 & 3,07 & 2,63 & 2,62 & 2,64 \\
\hline 8 & 3,00 & 2,99 & 3,00 & 2,80 & 3,02 & 3,00 & 2,75 & 3,02 & 2,82 \\
\hline 9 & 3,00 & 2,51 & 2,58 & 2,00 & 2,51 & 2,40 & 3,00 & 2,59 & 2,27 \\
\hline 10 & 1,50 & 1,96 & 1,91 & 2,20 & 1,94 & 2,20 & 1,38 & 1,93 & 1,91 \\
\hline 11 & 2,75 & 2,65 & 2,67 & 2,40 & 2,67 & 2,47 & 2,87 & 2,70 & 2,36 \\
\hline 12 & 2,50 & 2,22 & 2,24 & 2,20 & 2,14 & 2,40 & 2,50 & 2,21 & 2,36 \\
\hline 13 & 2,00 & 2,69 & 2,67 & 2,40 & 2,59 & 2,53 & 3,25 & 2,69 & 2,45 \\
\hline 14 & 2,75 & 2,82 & 2,84 & 2,60 & 2,76 & 2,93 & 3,00 & 2,80 & 2,91 \\
\hline $15 \mathrm{rec}$ & 3,25 & 2,69 & 2,70 & 3,00 & 2,84 & 2,53 & 2,38 & 2,70 & 2,82 \\
\hline 16 & 2,00 & 2,71 & 2,69 & 2,40 & 2,65 & 2,60 & 2,88 & 2,74 & 2,27 \\
\hline $17 \mathrm{rec}$ & 3,00 & 3,24 & 3,21 & 3,40 & 3,18 & 3,53 & 2,88 & 3,21 & 3,27 \\
\hline 18 & 2,75 & 2,50 & 2,51 & 2,60 & 2,57 & 2,33 & 2,50 & 2,56 & 2,27 \\
\hline 19 & 2,50 & 2,54 & 2,54 & 2,60 & 2,43 & 3,00 & 2,38 & 2,54 & 2,55 \\
\hline $20 \mathrm{rec}$ & 2,50 & 2,50 & 2,48 & 2,80 & 2,59 & 2,13 & 2,63 & 2,54 & 2,27 \\
\hline 21 & 2,75 & 3,26 & 3,22 & 3,40 & 3,12 & 3,27 & 3,87 & 3,28 & 3,00 \\
\hline 22 & 1,75 & 2,71 & 2,69 & 2,20 & 2,57 & 3,00 & 2,50 & 2,66 & 2,64 \\
\hline $23 \mathrm{rec}$ & 3,50 & 3,41 & 3,42 & 3,40 & 3,45 & 3,33 & 3,38 & 3,41 & 3,45 \\
\hline 24 & 3,50 & 2,87 & 2,93 & 2,60 & 2,88 & 2,87 & 3,13 & 2,95 & 2,64 \\
\hline 25 & 3,25 & 2,68 & 2,76 & 2,00 & 2,67 & 2,80 & 2,75 & 2,75 & 2,45 \\
\hline 26 & 2,75 & 3,12 & 3,10 & 3,00 & 3,16 & 2,87 & 3,13 & 3,15 & 2,82 \\
\hline 27 & 2,00 & 2,79 & 2,81 & 2,00 & 2,69 & 2,80 & 3,00 & 2,80 & 2,45 \\
\hline 28 & 3,00 & 2,72 & 2,75 & 2,60 & 2,73 & 2,67 & 2,88 & 2,70 & 2,91 \\
\hline 29 & 2,50 & 2,91 & 2,90 & 2,80 & 2,90 & 2,87 & 2,88 & 2,93 & 2,64 \\
\hline 30 & 2,50 & 2,60 & 2,61 & 2,40 & 2,63 & 2,53 & 2,50 & 2,54 & 2,91 \\
\hline 31 & 2,25 & 2,96 & 2,93 & 2,80 & 2,96 & 2,93 & 2,63 & 2,93 & 2,82 \\
\hline 32 & 2,50 & 2,82 & 2,81 & 2,80 & 2,86 & 2,73 & 2,63 & 2,84 & 2,64 \\
\hline 33 & 1,75 & 2,84 & 2,79 & 2,60 & 2,76 & 2,93 & 2,63 & 2,75 & 2,91 \\
\hline 34 & 2,00 & 2,50 & 2,54 & 1,60 & 2,53 & 2,47 & 2,13 & 2,44 & 2,64 \\
\hline 35 & 1,75 & 2,24 & 2,21 & 2,20 & 2,16 & 2,40 & 2,13 & 2,23 & 2,09 \\
\hline 36 & 2,75 & 2,85 & 2,85 & 2,80 & 2,86 & 2,80 & 2,87 & 2,84 & 2,91 \\
\hline 37 & 2,25 & 2,97 & 2,93 & 3,00 & 2,98 & 3,00 & 2,50 & 3,00 & 2,55 \\
\hline 38 & 3,00 & 2,60 & 2,63 & 2,60 & 2,59 & 2,93 & 2,25 & 2,56 & 3,00 \\
\hline 39 & 2,50 & 2,44 & 2,49 & 1,80 & 2,39 & 2,67 & 2,38 & 2,49 & 2,18 \\
\hline 40 & 2,00 & 2,76 & 2,75 & 2,40 & 2,63 & 2,87 & 3,00 & 2,72 & 2,73 \\
\hline
\end{tabular}

Tabla 3. Valores estadísticos medios en los ítems según cada categoría de comparación 
Con respecto a la relación entre el sexo y las ideas y actitudes sobre la integración universitaria de los estudiantes con discapacidad, se puede apreciar cómo las opiniones de las mujeres son más favorables significativamente a lo expresado en los siguientes ítems del cuestionario $(\mathrm{p}<0,05$, prueba $\mathrm{U}$ de Mann-Whitney para muestras independientes):

-Ítem 22: Se incrementan los esfuerzos económicos para la atención a los estudiantes con discapacidad (sig.: 0,024).

-Ítem 27: Los estudiantes, especialmente aquellos que presentan alguna discapacidad, conocen las competencias que se exigirán en cada titulación/carrera antes de formalizar su matrícula (sig.: 0,005), estando el $100 \%$ de los hombres en desacuerdo (sig.: 0,012).

-Ítem 33: Existe una adaptación de la infraestructura de la universidad para las diversas discapacidades (sig.: 0,032).

De igual manera, según los resultados obtenidos, los alumnos que han recibido anteriormente algún tipo de información por parte de la universidad de Bolonia sobre la presencia de estudiantes con discapacidad en sus aulas se manifiestan más en desacuerdo, de manera significativa, hacia las siguientes aseveraciones $(\mathrm{p}<0,05$, prueba U de Mann-Whitney para muestras independientes):

-Ítem 25: Las pruebas o exámenes en la universidad contemplan los recursos y adaptaciones necesarias para dar respuesta a las necesidades de los estudiantes con discapacidad (sig.: 0,032).

-Ítem 27: Los estudiantes, especialmente aquellos que presentan alguna discapacidad, conocen las competencias que se exigirán en cada titulación/carrera antes de formalizar su matrícula (sig.: 0,009).

$\mathrm{Si}$ analizamos ahora las distribuciones de datos según los grupos de edad, apreciamos que el grupo de edades comprendidas entre 22 y 26 años, destaca en los ítems 7 (los objetivos y contenidos son idénticos -los mismos- para todos los estudiantes) y 19 (la universidad dispone de los medios para solventar cualquier tipo de adaptación en el acceso al currículum para estos estudiantes) (sig.: 0,046 y 0,012, respectivamente; prueba $U$ de Mann-Whitney para muestras independientes).

Por otra parte, el grupo de edad más avanzada (edades comprendidas entre 27 y 41) destaca en el ítem 21 (la universidad dispone de los medios para solventar cualquier tipo de adaptación en el acceso al currículum para estos estudiantes) (sig.: 0,010; prueba U de Mann-Whitney para muestras independientes).

Por último, atendiendo a la variable de si han tenido o no compañeros con discapacidad, hemos encontrado que no existen diferencias significativas entre alumnos en relación con las ideas y actitudes hacia la integración de estudiantes universitarios con discapacidad. 


\section{Interpretación de observaciones}

Como último aspecto a analizar, deseamos reseñar algunos comentarios de entre los muchos que se expresaron por parte de los encuestados, los cuales destacan por su carácter representativo e ilustran el sentir del colectivo de estudiantes de la universidad de Bolonia hacia el objeto del estudio que presentamos:

- "Es importante que los docentes estemos preparados y que haya atención a la discapacidad".

- "No he tenido compañeros con discapacidad en la universidad pero sí en la escuela primaria. Contesto en función de dicha experiencia".

- "No sé realmente cómo afronta mi facultad dicha necesidad, respondo en función de lo que considero".

- Etc.

En este sentido apreciamos que se considera importante la preparación del colectivo de futuros docentes hacia esta temática, así como su adecuada implantación en la universidad, si bien reconocen que no han tenido, en su mayoría, ocasión de compartir espacios de aprendizaje con alumnos con discapacidad en dicho nivel educativo dado el reducido número que acceden al mismo (no así en los anteriores). Por último, se reconoce una falta de información y formación de esta problemática, sus necesidades y la manera de afrontarla por parte de la institución universitaria hacia el conjunto de la comunidad educativa.

\section{Discusión y conclusiones}

Tras los análisis realizados y atendiendo a los resultados descriptivos generales, podemos concluir que los estudiantes que cursan el grado de educación primaria en la universidad de Bolonia poseen unas ideas y actitudes favorables hacia la integración educativa y social de los estudiantes con discapacidad.

Con respecto al análisis según categorías podemos concluir que prevalece la categoría "aceptación" entre el conjunto de las planteadas, ya que hemos detectado que existe una percepción muy favorable por parte de los estudiantes de la universidad de Bolonia hacia el alumnado con discapacidad y su integración educativa y social. Sobre todo cuando consideran que la presencia de estudiantes con discapacidad en el aula no obstaculiza el nivel académico general (ítem 23) ni limita el aprendizaje del alumnado (ítem 6).

Siguiendo esta línea, y en relación con la categoría referida a las ideas y actitudes que tienen los estudiantes sobre los servicios universitarios para favorecer la integración del alumnado con discapacidad, podemos destacar que la mayoría de los encuestados opinan que en la universidad se cumple la normativa que contempla los derechos y deberes en relación con la integración de dicho tipo de alumnado (ítem 31).

De manera global, si contrastamos los resultados del presente estudio (universidad de Bolonia) con los obtenidos en investigaciones similares llevadas a cabo con el 
mismo instrumento de recogida de datos (o levemente adaptado en el caso del contexto mexicano) y tipo de muestra en la universidad de Almería (España) en 2009 y en la universidad de Tlaxcala (México) en 2011, apreciamos lo siguiente (véase tabla 3):

\begin{tabular}{ccc}
\hline $\begin{array}{c}\text { CONTEXTO } \\
\text { (ALUMNADO) } \\
\text { Número participantes }\end{array}$ & $\begin{array}{c}\text { MEDIA DE } \\
\text { RESULTADOS A FAVOR } \\
\text { DE LA INTEGRACIÓN }\end{array}$ & $\begin{array}{c}\text { DESVIACIÓN TÍPICA } \\
\text { DE RESULTADOS }\end{array}$ \\
\hline $\begin{array}{c}\text { ALMERÍA (ESPAÑA) } \\
2009\end{array}$ & 2,27 & 0,65 \\
544 & 2,81 & 0,79 \\
\hline $\begin{array}{c}\text { TLAXCALA (MÉXICO) } \\
2011\end{array}$ & 2,72 & 0,77 \\
\hline $\begin{array}{c}\text { BOLONIA (ITALIA) } \\
2012\end{array}$ & & \\
272 & & \\
\hline
\end{tabular}

Tabla 3. Resultados descriptivos según contextos

Como vemos, los resultados en el contexto italiano son similares a los estudios anteriores, si bien presentan un leve incremento con relación a los obtenidos en Almería (2009) y algo más de variabilidad que éstos respecto a la media. En cualquier caso, el contexto mexicano sigue predominando respecto al europeo con relación a la actitud receptiva del alumnado de cara a la implantación de las medidas integradoras del alumnado con discapacidad en la universidad.

Si procedemos a comparar en mayor detalle los datos de la investigación que presentamos con los obtenidos en el estudio similar realizado en la universidad de Almería (Sánchez, 2009), anotamos lo siguiente:

a) Ambas comunidades estudiantiles manifiestan similar tendencia en cuanto a mostrar su acuerdo con el hecho de:

- Fomentar actitudes favorecedoras en los diferentes colectivos de alumnado con discapacidad.

- Existir una predisposición general para facilitar el acceso a la universidad de los estudiantes con discapacidad exigiendo a la institución universitaria que ponga los medios materiales y personales para su integración.

- Deber incrementarse los esfuerzos económicos para mejorar la atención educativa que reciben así como que se tienen que fomentar actitudes favorables hacia ellos.

- No flexibilizar a la hora de exigirles la adquisición de las competencias propias de sus carreras.

- Tener las mismas oportunidades y posibilidades de promoción que el resto de sus compañeros, defendiendo el principio de igualdad de oportunidades. 
b) Sin embargo, se aprecia cierta discrepancia con relación a los siguientes aspectos:

- Existir una unidad central que coordine y asesore a estudiantes con discapacidad, al profesorado y al personal de administración y servicios (mayor predisposición en Almería).

- Considerar beneficioso la existencia en las aulas de estudiantes con discapacidad para todos los universitarios (mayor predisposición en Bolonia).

- Clara disposición a formarse para facilitar la estancia de los estudiantes con discapacidad (mayor predisposición en Almería).

Igualmente, analizando comparativamente ambos estudios, podemos señalar que, si bien en la universidad de Bolonia hemos encontrado algunas diferencias significativas entre las opiniones de los encuestados en función del sexo, edad o el hecho de haber recibido o no información en la universidad acerca de la presencia de compañeros con discapacidad, en el contexto almeriense dichas discrepancias entre subgrupos no fueron significativas entre el alumnado de magisterio, siendo en ambos estudios muy reducido el número de estudiantes que manifiestan haber recibido algún tipo de formación relacionada con la temática.

Sin embargo, con relación al análisis comparativo de ideas y actitudes entre los subgrupos relacionados con la variable tener o haber tenido en clase compañeros o compañeras con discapacidad, se desprende, para ambos contextos (Almería y Bolonia) que no hay diferencias significativas ni en las actitudes ni en las ideas manifestadas por los estudiantes de las titulaciones de magisterio de sendas universidades.

Tras todo lo comentado, y volviendo al contexto objeto de nuestra investigación, podemos afirmar en términos generales que, a pesar de que los planteamientos institucionales avanzan teóricamente a buen ritmo, la integración del colectivo de estudiantes con discapacidad en la educación superior es una tarea que se muestra aún inconclusa en la universidad de Bolonia (al igual que en los otros contextos mencionados), no desdeñando la apreciable tendencia favorable en dicho contexto, la cual resulta posiblemente explicable por la larga y amplia trayectoria de integración escolar que se vive en Italia.

Según los informes que hemos analizado, y con objeto de verificar la representatividad de los datos, quedaría por analizar la situación que presentan ante la temática aquí tratada otras universidades españolas o europeas, así como las causas de dicha realidad y los planteamientos concretos que están llevándose a cabo para afrontarla, así como hacer propuestas de mejora sobre la integración educativa y social de los estudiantes con necesidades educativas especiales asociadas a una discapacidad.

En este sentido, consideramos imprescindible el desarrollo de estándares y guías de buenas prácticas en todos aquellos aspectos de la vida académica que puedan conducir a situaciones de desigualdad para un estudiante con discapacidad (véase, por ejemplo, Alonso y Díez, 2008; Dukes, 2001, 2006; Díez y otros, 2011). Así como la utilización generalizada de las tecnologías de la información y la comunicación (TIC) en las aulas universitarias, siendo éstas un elemento favorecedor de la integración de estudiantes 
con discapacidad en el sistema universitario, ya que la formación con nuevas tecnologías permite superar barreras físicas, temporales, o de capacidades cognitivas que se pueden encontrar en determinados perfiles de discapacidad.

Con todo lo aquí expuesto, queremos manifestar que el avance hacia el Espacio Europeo de Educación Superior requiere, en este sentido, de nuevos escenarios conjuntos y puntos de encuentro entre la comunidad universitaria y el movimiento asociativo de la discapacidad, para lo cual el análisis de la realidad desde planteamientos comparativos y la propuesta de actuaciones conjuntas resultarán clave en la implantación de medidas eficaces de cara a la integración de este tipo de alumnado en las instituciones de educación superior y en la sociedad en general.

\section{Referencias bibliográficas}

AINSCOW, M. (2001). Desarrollo de escuelas inclusivas. Madrid: Ed. Narcea.

ALONSO, A. \& DÍEZ, E. (2008). Universidad y Discapacidad: indicadores de buenas prácticas y estándares de actuación para programas y servicios. Siglo Cero, 39, 8298.

ARANA, J. M., GARCÍA, J.J. \& RODRÍGUEZ, S. (2008). Los Servicios de Atención Psicológica en la universidad española: Análisis crítico. Revista de Psicología General y Aplicada, 61, 265-284.

ARIAS-MARTÍNEZ, B. (1994). Evaluación de actitudes hacia la integración de alumnos con necesidades educativas especiales. Tesis doctoral. Departamento de Psicología, Universidad de Salamanca.

AVRAMIDIS, E, BAYLISS, P. \& BURDEN, R. (2000). Student teachers' attitudes towards the inclusion of children with special educational needs in the ordinary school. Teaching and teacher education, 16, 277-293.

BAUSELA, E. (2004). La función orientadora en el marco de la Universidad. EduPsykhé, 3, 109-120.

BUENO, A. (2010). Una mirada ilusionada al futuro de los Servicios de Apoyo a la Discapacidad en la Universidad. Buenas prácticas de apoyo a la discapacidad en la universidad. En: Bueno, A. (coord.), Buenas prácticas durante los estudios universitarios en la recepción de la información (pp. 10-33). Alicante: Editorial CEE Limencop.

CASTEJÓN, L. (2004). Percepciones y actitudes sobre el alumno tartamudo en Educación Primaria. Tesis Doctoral, Universidad de Oviedo.

CENTER, Y. \& WARD, J. (1987). Teachers' attitudes towards the integration of disabled children into regular schools. Exceptional Child, 34, 41-56.

CLOUGH, P. \& LINDSAY, G. (1991). Integrationand the Support Service. Slough: NFER 
DE ANNA, L. (2010). La integración escolar en Italia. Revista de Educación Inclusiva, 3 (1), 111-117.

DE MAURO, T. (2004). La cultura degli italiani. Bari: Laterza.

DENDRA, M. R., DURÁN, B. R. \& VERDUGO, M. A. (1991). Estudio de las variables que afectan a las actitudes de los maestros hacia la integración escolar de niños con necesidades especiales. Psicopedagogía terapéutica, 47-88.

DÍAZ, F.A. (2000). Importancia de la orientación educativa en la atención a la diversidad de alumnos/as con necesidades educativas especiales. Narración de experiencias. En: Salmerón, V. \& López, V.L. (Coords.), Orientación Educativa en las Universidades (pp. 269-272). Granada: Grupo Editorial Universitario.

DÍEZ, E., ALONSO, A., VERDUGO, M.A., CAMPO, M., SANCHO, I., SÁNCHEZ, S., CALVO, I. \& MORAL, E. (2011). Espacio Europeo de Educación Superior: estándares e indicadores de buenas prácticas para la atención a estudiantes universitarios con discapacidad. Salamanca: INICO.

DÍEZ, E. \& VERDUGO, M. A. (1997). La teleformación como alternativa a la formación presencial para personas con discapacidad. En: Verdugo, M. A. et al. (comps.), Actas de las II Jornadas de Investigación sobre Personas con Discapacidad (pp. 311-318). Salamanca: Gráicas Varona.

DUKES, L. L. (2001). The process: Development of AHEAD Program Standards. Journal of Postsecondary Education and Disability, 14, 62-80.

DUKES, L. L. (2006). The Process: Development of the revised AHEAD Program Standards and Performance Indicators. Journal of Postsecondary Education and Disability, 19, 5-15.

ECHES, S. \& OCHOA, T. (2005). Students with disabilities: Transitioning from High School to higher education. American Secondary Education, 33, 6-20.

FOX, D.J. (1981). El proceso de investigación en educación. Pamplona: Eunsa.

GOETZ, J.P. \& LECOMPTE, M.D. (1988). Etnografia y diseño cualitativo en investigación educativa. Madrid: Morata.

GÓMEZ, V. \& INFANTE, M. (2004). Actitudes de los estudiantes de educación hacia la integración de personas con discapacidad y hacia la educación multicultural. Cultura y Educación, 16 (4), 371-383.

GRANADOS, A. (2000). ¿Tiene cabida la diversidad en la Universidad?. En: Salmerón, V. \& López, V.L. (coords.), Orientación Educativa en las Universidades (pp. 144-147). Granada: Grupo Editorial Universitario.

GUZMÁN ZAMORA. J. \& SÁNCHEZ PALOMINO, A. (2011). La integración Educativa y Social de Estudiantes con Discapacidad en las Instituciones de Educación Superior del Estado de Tlaxcala (México). Tlaxcala: Universidad de Tlaxcala. 
HEGARTY, S., HODGSON A. \& CLUMIES-ROSS, L. (1988). Aprender juntos: La integración escolar. Madrid: Ed. Morata.

HODDER, I. (2000). The interpretation of documents and material culture. En: Denzin, N. K. \& Lincoln, S. (eds.), Handbook of Qualitative Research (pp. 703-717). London: Sage Publications

ISTAT (2009). La disabilità in Italia. Il cuadro Della statistica ufficiale. Roma: Istat, Sistema Statistico Nazionale, Istituto Nazionale de Statistica.

JARVIS, K.C. \& FRENCH, R. (1990). Attitudes of physical educators toward the integration of handicapped students. Perceptual and Motor Skills, 70, 899-902.

KONUR, O. (2006). Teaching disabled students in higher education. Teaching in Higher Education, 11, 351- 363.

LEGGE 5 febbraio 1992, n. 104, Legge-quadro per l'assistenza, l'integrazione sociale e i diritti delle persone handicappate.

LEGGE 4 agosto 1977, n. 517, Norme sulla valutazione degli alunni e sull'abolizione degli esami di riparazione nonché altre norme di modifica dell'ordinamento scolastico.

LEGGE 28 gennaio 1999, n. 17, Integrazione e modifica della leggequadro 5 febbraio 1992, n. 104, per l'assistenza, l'integrazione sociale e i diritti delle persone handicappate.

MANGAS MARTÍN, A. (2005). La constitución europea. Revista del Ministerio de Trabajo y Asuntos Sociales, 57, 570-581.

MORENO, F.J., RODRÍGUEZ, I.R., SALDAÑA, D. \& AGUILERA, A. (2006) Actitudes ante la discapacidad en el alumnado universitario matriculado en materias afines. Revista Iberoamericana de Educación, 40, 5-25.

PAVONE, M. (2007). La dirección escolar en Italia. Revista Española de Educación Comparada, 13, 53-82.

RODRÍGUEZ GÓMEZ, G., GIL FLORES, J. \& GARCÍA JIMÉNEZ, E. (1996). Metodología de la Investigación Cualitativa. Málaga: Ediciones Aljibe.

ROSENVINGE, A., ORTEGA M. \& CAMPO C. (1991). La integración en la escuela infantil. Madrid: Ed. Alameda.

RUBIRALTA, M. (2012). Las políticas sobre la discapacidad en el sistema universitario español. Madrid: MEC.

SÁENZ, O. (1990). Actitudes de los profesores ante la integración del niño discapacitado en la escuela ordinaria. Revista Interuniversitaria de Formación del Profesorado, 8, 135-150.

SÁNCHEZ PALOMINO, A. (2009). La integración educativa y social de los estudiantes con discapacidad en la universidad de Almería. Almería: Universidad de Almería. 
SÁNCHEZ PALOMINO, A. (2011). La Universidad de Almería ante la integración educativa y social de los estudiantes con discapacidad: Ideas y actitudes del personal docente e investigador. Revista de Educación, 354, 575-603.

SCIACCA, S., SCIUTO, S., SIGNORINO, L. VENUTO, A.S. \& NICOTRA, G. (2008). Sistema educativo italiano: legislación para atender a alumnos con discapacidad. Doces, 1, 1-4.

STAINBACK, S. \& STAINBACK, W. (1999). Aulas inclusivas. Madrid. Ed. Narcea.

SURIÁ MARTÍNEZ, R. (2011). Análisis comparativo sobre las actitudes de los estudiantes hacia sus compañeros con discapacidad. Electronic Journal of Research in Educational Psychology, 9 (1), 197-216.

TRAINITO, G. (2003). La scuola italiana in Europa: quale valutazione per migliorare l'offerta. Quaderni degli Annali dell'Istruzione, 102, 6-16.

VEGA, A. (2000). La educación ante la discapacidad. Hacia una respuesta social de la escuela. Valencia: Ed. Culturals Valencianes S.A.

VERDUGO, M. A., ARIAS, B. \& JENARO, C. (1994). Actitudes hacia las personas con minusvalía. Madrid: Ministerio de Asuntos Sociales, Instituto Nacional de Servicios Sociales.

\section{Correspondencia con los autores}

Antonio LUQUE DE LA ROSA

Departamento de Educación. Universidad de Almería

Carretera de Sacramento, $\mathrm{s} / \mathrm{n}$

04120 La Cañada de San Urbano (Almería)

e mail: aluque@ual.es

\section{Rafaela GUTIÉRREZ CÁCERES}

Departamento de Educación. Universidad de Almería

Carretera de Sacramento, s/n

04120 La Cañada de San Urbano, 04120 (Almería)

e mail: rcaceres@ual.es 


\section{ANEXO 1}

Cuestionario dirigido a estudiantes de la Universidad de Bolonia 2011/12

\section{CUESTIONARIO PARA LOS ESTUDIANTES DE LA UNIVERSIDAD DE BOLONIA 2011/2012}

Con este cuestionario, pretendemos conocer las opiniones del alumnado Erasmus tras visitar la comunidad educativa de la Universidad de Bolonia hacia la integración educativa y social de los estudiantes con discapacidad en dicha universidad.

\section{GRACIAS POR TU COLABORACIÓN}

\section{DATOS}

Edad

Género: Hombre Mujer Carrera/ Titulación .Curso

Tengo, o he tenido, en Bolonia, en clase a estudiantes con discapacidad:

SÍ $\square \quad \mathrm{Nq} \square$

En caso afirmativo indique el tipo: (Tache con una $\mathrm{X}$ la respuesta elegida)

Física $\square$ Psíquica $\square$ Auditiva $\square \quad$ Visual

He recibido algún tipo de información en la Universidad de Bolonia acerca de la presencia de compañeros o compañeras con discapacidad:

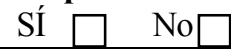

\section{CUESTIONARIO}

(Tache con una x la puntuación más adecuada en función del grado de acuerdo-desacuerdo):

1.- Totalmente en desacuerdo

3.- De acuerdo

2.- En desacuerdo

4.- Totalmente de acuerdo

"En calidad de estudiante de la Universidad de Bolonia, pienso que..."

\begin{tabular}{|c|l|l|l|l|l|l|l|l|}
\hline 1 & $\begin{array}{l}\text { Se facilita el acceso de los estudiantes con discapacidad a la } \\
\text { Universidad }\end{array}$ & 1 & 2 & 4 \\
\hline 2 & Es normal tener compañeros y compañeras con discapacidad en clase & 1 & 2 & 3 & 4 \\
\hline 3 & $\begin{array}{l}\text { A los estudiantes con discapacidad de Bolonia les cuesta más terminar } \\
\text { una carrera al no disponer de medios adaptados }\end{array}$ & 1 & 2 & 3 & 4 \\
\hline 4 & $\begin{array}{l}\text { Tengo más dificultad para relacionarme con compañeros y } \\
\text { compañeras que presentan alguna discapacidad }\end{array}$ & 1 & 3 & 4 \\
\hline 5 & $\begin{array}{l}\text { Me gusta relacionarme con compañeros discapacitados a los que } \\
\text { pueda serle útil }\end{array}$ & 1 & 2 & 3 & 4 \\
\hline 6 & $\begin{array}{l}\text { La existencia en las aulas de estudiantes con discapacidad dificulta el } \\
\text { aprendizaje }\end{array}$ & 1 & 2 & 3 & 4 \\
\hline 7 & $\begin{array}{l}\text { Los objetivos y contenidos son idénticos (los mismos) para todos los } \\
\text { estudiantes }\end{array}$ & 1 & 2 & 3 & 4 \\
\hline 8 & $\begin{array}{l}\text { Se fomentan actitudes favorables hacia la integración de estudiantes } \\
\text { con discapacidad }\end{array}$ & 1 & 2 & 3 & 4 \\
\hline
\end{tabular}




\begin{tabular}{|c|c|c|c|c|c|}
\hline 9 & $\begin{array}{l}\text { Se facilita a estos estudiantes el acceso a los contenidos y a la } \\
\text { evaluación según sus necesidades }\end{array}$ & 1 & 2 & 3 & 4 \\
\hline 10 & $\begin{array}{l}\text { He recibido algún asesoramiento o formación para facilitar su estancia } \\
\text { en la Universidad }\end{array}$ & 1 & 2 & 3 & 4 \\
\hline 11 & $\begin{array}{l}\text { Existe una unidad central que coordina y asesora a estudiantes con } \\
\text { discapacidad, al profesorado implicado en su formación y al personal } \\
\text { de administración y servicios }\end{array}$ & 1 & 2 & 3 & 4 \\
\hline 12 & $\begin{array}{l}\text { Se informa a los estudiantes de la existencia en sus aulas de } \\
\text { compañeros y compañeras con discapacidad }\end{array}$ & 1 & 2 & 3 & 4 \\
\hline 13 & $\begin{array}{l}\text { Existe en la Universidad profesorado especializado para estos } \\
\text { estudiantes }\end{array}$ & 1 & 2 & 3 & 4 \\
\hline 14 & $\begin{array}{l}\text { La Universidad pone los medios para que los estudiantes con } \\
\text { discapacidad participen en la vida universitaria como el resto de sus } \\
\text { compañeros y compañeras }\end{array}$ & 1 & 2 & 3 & 4 \\
\hline 15 & $\begin{array}{l}\text { En la Universidad existen grupos específicos para los estudiantes con } \\
\text { discapacidad }\end{array}$ & 1 & 2 & 3 & 4 \\
\hline 16 & $\begin{array}{l}\text { Existen personal o recursos en la Universidad, fruto de convenios de } \\
\text { colaboración con Asociaciones e Instituciones Públicas y/o Privadas } \\
\text { para mejorar la atención a los estudiantes con discapacidad }\end{array}$ & 1 & 2 & 3 & 4 \\
\hline 17 & $\begin{array}{l}\text { La presencia en las aulas de estudiantes con discapacidad supone } \\
\text { problemas y dificultades }\end{array}$ & 1 & 2 & 3 & 4 \\
\hline 18 & $\begin{array}{l}\text { iste una normativa específica que regula la atención a los } \\
\text { udiantes con discapacidad en la Universidad de Bolonia. }\end{array}$ & 1 & 2 & 3 & 4 \\
\hline 19 & $\begin{array}{l}\text { La Universidad dispone de los medios para solventar cualquier tipo de } \\
\text { adaptación en el acceso al currículum para estos estudiantes }\end{array}$ & 1 & 2 & 3 & 4 \\
\hline 20 & $\begin{array}{l}\text { Se tiene con ellos más flexibilidad a la hora de exigirles la adquisición } \\
\text { de las competencias propias de las titulaciones/carreras }\end{array}$ & 1 & 2 & 3 & 4 \\
\hline 21 & $\begin{array}{l}\text { La presencia en las aulas de estudiantes con discapacidad beneficia a } \\
\text { todos y a todas }\end{array}$ & 1 & 2 & 3 & 4 \\
\hline 22 & $\begin{array}{l}\text { Se incrementan los esfuerzos económicos para la atención a los } \\
\text { estudiantes con discapacidad }\end{array}$ & 1 & 2 & 3 & 4 \\
\hline 23 & $\begin{array}{l}\text { La presencia en las aulas de estudiantes con discapacidad produce un } \\
\text { descenso del nivel académico generalizado }\end{array}$ & 1 & 2 & 3 & 4 \\
\hline 24 & $\begin{array}{l}\text { Los estudiantes con discapacidad tienen las mismas oportunidades y } \\
\text { posibilidades de promoción que los demás universitarios y } \\
\text { universitarias }\end{array}$ & 1 & 2 & 3 & 4 \\
\hline 25 & $\begin{array}{l}\text { Las pruebas o exámenes en la universidad contemplan los recursos y } \\
\text { adaptaciones necesarias para dar respuesta a las necesidades de los } \\
\text { estudiantes con discapacidad }\end{array}$ & 1 & 2 & 3 & 4 \\
\hline 26 & $\begin{array}{l}\text { La titulación alcanzada por los estudiantes con discapacidad supone el } \\
\text { garante del conjunto de competencias para su ejercicio profesional }\end{array}$ & 1 & 2 & 3 & 4 \\
\hline
\end{tabular}




\begin{tabular}{|c|c|c|c|c|c|}
\hline 27 & $\begin{array}{l}\text { Los estudiantes, especialmente aquellos que presentan alguna } \\
\text { discapacidad, conocen las competencias que se exigirán en cada } \\
\text { titulación/carrera antes de formalizar su matrícula }\end{array}$ & 1 & 2 & 3 & \\
\hline 28 & $\begin{array}{l}\text { La Universidad desarrolla un Plan de Acogida para los estudiantes con } \\
\text { discapacidad }\end{array}$ & 1 & 2 & 3 & \\
\hline 29 & $\begin{array}{l}\text { Existe comunicación entre compañeros y compañeras como apoyo y } \\
\text { ayuda a estudiantes con discapacidad }\end{array}$ & 1 & 2 & 3 & \\
\hline 30 & $\begin{array}{l}\text { Existe una reserva de plaza en cada titulación para alumnos con } \\
\text { discapacidad, así como en residencias y colegios mayores. }\end{array}$ & 1 & 2 & 3 & \\
\hline 31 & $\begin{array}{l}\text { Se fomenta el cumplimiento de normativas universitarias que } \\
\text { determinan los derechos y deberes de las personas con discapacidad; }\end{array}$ & 1 & 2 & 3 & 4 \\
\hline 32 & $\begin{array}{l}\text { Se promueve la concienciación de la comunidad educativa acerca de } \\
\text { los servicios disponibles para los alumnos con discapacidad. }\end{array}$ & 1 & 2 & 3 & \\
\hline 33 & ra de la universidad para las & 1 & 2 & 3 & 4 \\
\hline 34 & $\begin{array}{l}\text { La metodología (espacios, mobiliarios, materiales, horarios, etc.) } \\
\text { consideran la atención a la diversidad por discapacidad }\end{array}$ & 1 & 2 & 3 & 4 \\
\hline 35 & $\begin{array}{l}\text { Los planes de estudio consideran un considerable número de créditos } \\
\text { para formar a los maestros en la atención a las discapacidades en el } \\
\text { ámbito escolar }\end{array}$ & 1 & 2 & 3 & \\
\hline 36 & $\begin{array}{l}\text { isten planes/itinerarios específicos en el grado de especialización en } \\
\text { ención al alumnado con discapacidad. }\end{array}$ & 1 & 2 & 3 & \\
\hline 37 & $\begin{array}{l}\text { Las diversas asignaturas se enfocan para promover en el futuro } \\
\text { docente la adaptación a situaciones de alumnado con discapacidad }\end{array}$ & 1 & 2 & 3 & 4 \\
\hline 38 & $\begin{array}{l}\text { Existen intérpretes de lengua de signos en las clases que tienen } \\
\text { alumnado con sordera. }\end{array}$ & 1 & 2 & 3 & \\
\hline 39 & $\begin{array}{l}\text { Existen planes/itinerarios específicos en el grado de especialización en } \\
\text { atención al alumnado con problemas de lenguaje y audición }\end{array}$ & 1 & 2 & J & 4 \\
\hline 40 & $\begin{array}{l}\text { Los servicios culturales de la comunidad están adaptados para el } \\
\text { alumnado con discapacidad. }\end{array}$ & 1 & 2 & & 4 \\
\hline
\end{tabular}

\section{OBSERVACIONES QUE DESEE FORMULAR}

